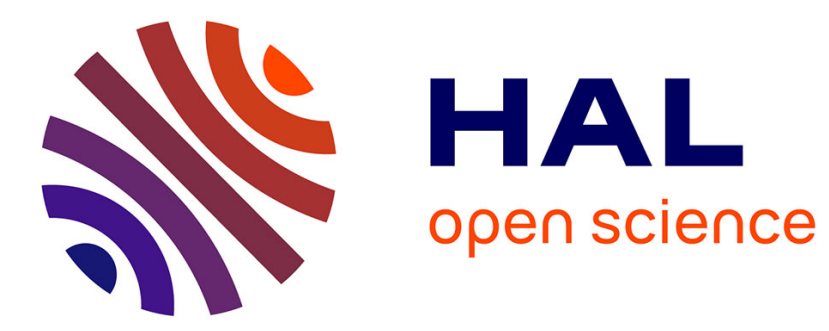

\title{
Model for Heat and Mass Transfer in Freeze-Drying of Pellets
}

Ioan-Cristian Trelea, Stephanie Passot, Michele Marin, Fernanda Fonseca

\section{To cite this version:}

Ioan-Cristian Trelea, Stephanie Passot, Michele Marin, Fernanda Fonseca. Model for Heat and Mass Transfer in Freeze-Drying of Pellets. Journal of Biomechanical Engineering, 2009, 131 (7), pp.074501. 10.1115/1.3142975 . hal-01536947

\section{HAL Id: hal-01536947 \\ https://hal-agroparistech.archives-ouvertes.fr/hal-01536947}

Submitted on 16 Jun 2017

HAL is a multi-disciplinary open access archive for the deposit and dissemination of scientific research documents, whether they are published or not. The documents may come from teaching and research institutions in France or abroad, or from public or private research centers.
L'archive ouverte pluridisciplinaire HAL, est destinée au dépôt et à la diffusion de documents scientifiques de niveau recherche, publiés ou non, émanant des établissements d'enseignement et de recherche français ou étrangers, des laboratoires publics ou privés. 


\title{
Model for Heat and Mass Transfer in Freeze-Drying of Pellets
}

Ioan Cristian Trelea*, Stéphanie Passot, Michèle Marin and Fernanda Fonseca

UMR 782 Génie et Microbiologie des Procédés Alimentaires, AgroParisTech - INRA

1 av. Lucien Brétignières, 78850 Thiverval-Grignon, France

*corresponding author : $\quad$ cristian.trelea@agroparistech.fr

phone : +33130815490, fax : +33130815597

\begin{abstract}
Background. Lyophilizing frozen pellets, and especially spray freeze-drying receive growing interest. To design efficient and safe freeze-drying cycles, local temperature and moisture content in the product bed have to be known, but are difficult to measure in industry. Mathematical modeling of heat and mass transfer helps determining local freeze-drying conditions and predicting effects of operation policy, equipment and recipe changes on drying time and product quality.
\end{abstract}

Method of approach. Representative pellets situated at different positions in the product slab were considered. One dimensional transfer in the slab and radial transfer in the pellets was assumed. Coupled heat and vapor transfer equations between the temperature-controlled shelf, the product bulk, the sublimation front inside the pellets and the chamber were established and solved numerically.

Results. The model was validated based on bulk temperature measurement performed at two different locations in the product slab and on partial vapor pressure measurement in the freeze-drying chamber. Fair agreement between measured and calculated values was found. In contrast, a previously developed model for compact product layer was found inadequate to describe freeze-drying of pellets.

Conclusions. The developed model represents a good starting basis for studying freezedrying of pellets. It has to be further improved and validated for a variety of product types and freeze-drying conditions (shelf temperature, total chamber pressure, pellet size, slab thickness, etc.). It could be used to develop freeze-drying cycles based on product quality criteria such as local moisture content and glass transition temperature. 
Model for heat and mass transfer in freeze-drying of pellets

Keywords: lyophilization, freeze-drying, dynamic model, pellets

\section{Introduction}

Freeze-drying (or lyophilization) is widely used to preserve and ensure long-term stability of bioactive ingredients such as proteins, micro-organisms and vaccines. But it remains a timeand energy-consuming process. The process involves freezing of the aqueous solution containing the active ingredient, followed by primary drying to remove ice by sublimation and, finally, secondary drying to remove unfrozen or sorbed water [1]. The longest process part is usually the sublimation of ice from the frozen layer. The product temperature during primary drying, depending on the shelf temperature and the pressure, is a critical parameter. Too high temperature can result in product collapse (with potential degradation of the active ingredient), whereas a too low temperature will result in unnecessarily lengthy cycles [2-5].

A key parameter governing the relationship between the independent process variables (shelf temperature and pressure) and the dependent variables (product temperature) is the resistance of the dried product layer to mass transfer $[6,7]$. The product temperature normally increases during primary drying as the dried layer resistance increases with sublimation time, which could result in collapse of the product. An alternative to limit the impact of the dried layer resistance on the product temperature is to change the geometry of the frozen layer and to lyophilize a pellet layer creating a more open and porous structure.

Product stability depends on local temperature and moisture conditions encountered during freeze-drying [4]. Such local conditions can not be measured directly during the freeze-drying process in industrial situation, especially inside pellets. The developed heat and mass transfer model helps assessing local processing conditions in the bulk of the product slab and inside pellets, in order to assist freeze-drying cycle development and predict local product degradation risk at any location. The developed heat and mass transfer model is intended to be coupled to quality degradation models, with appropriate definitions of quality for each product type. 
Model for heat and mass transfer in freeze-drying of pellets

\section{Materials and methods}

\section{Product preparation}

A model medium composed of a mixture of $120 \mathrm{~g} / \mathrm{L}$ of sucrose (Prolabo, Paris, France) and of $180 \mathrm{~g} / \mathrm{L}$ of maltodextrin DE12 (Roquette, Lestrem, France) was immersed in liquid nitrogen to obtain frozen droplets with an average diameter of $2 \mathrm{~mm}$.

\section{Freeze-drying protocols}

The frozen pellets were placed in a tray and loaded onto the pre-cooled shelf (at $-45^{\circ} \mathrm{C}$ ) of a freeze-dryer pilot plant (SMH 15 model, Usifroid, Maurepas, France). Three product fill depths (25 mm, $35 \mathrm{~mm}$ and $45 \mathrm{~mm}$ ) were considered.

After a holding step at $-45^{\circ} \mathrm{C}$, the chamber was evacuated to a defined pressure value to initiate primary drying. Various combinations of shelf temperature (from $0^{\circ} \mathrm{C}$ to $45^{\circ} \mathrm{C}$ ) and chamber pressure (from $10 \mathrm{~Pa}$ to $40 \mathrm{~Pa}$ ) were applied during the primary drying step. When the end point of the sublimation was detected, the secondary drying step was initiated by increasing the shelf temperature. The sublimation end point was detected based on the partial vapor pressure drop in the chamber below $2 \mathrm{~Pa}$. The shelf temperature was either risen to $25^{\circ} \mathrm{C}$ when primary drying was performed at value lower than $25^{\circ} \mathrm{C}$ or maintained at the shelf temperature value applied during primary drying. The length of the secondary drying step was fixed at 11 hours. All cycles ramps were performed with a heating rate of $0.2^{\circ} \mathrm{C} / \mathrm{min}$.

Two thermocouples were placed in the layer of pellets, one at the bottom of the layer and the other in the middle of the layer. A moisture sensor (Parametrics Ltd, Shannon, Ireland) was used to monitor the partial vapor pressure in the chamber.

\section{Mathematical model for heat and mass transfer in a layer of pellets}

A schematic representation of the heat transfer during freeze-drying in a layer of pellets is shown in Figure 1. The heat is mainly supplied at the bottom of the product layer by the temperature-controlled shelf via the tray, by direct contact, radiation and conduction through the ambient gas [8]. To a lesser extent, the heat is also supplied to the top of the product by the freeze-drying chamber, mainly by radiation. The major part of the supplied heat is used for ice sublimation [9]. Unlike conventional freeze-drying of a solution filled in a tray or vial (compact product layer configuration), in the case of pellets there is no well-defined separation between the frozen part of the product at the bottom of the tray and the dry part at the top. Rather, sublimation occurs simultaneously in all pellets. The dry part is situated at the 
periphery of the pellet and the frozen part in its core. This is in contrast to the view adopted in a recent work [10], where the existence of a sublimation front situated inside the slab was considered, with all pellets situated above the front being dry and all pellets situated below being frozen. Unfortunately, no experimental validation of this modeling hypothesis is provided in that work.

To model the considered phenomena, the slab of pellets was divided in a number of virtual layers and heat transfer was assumed between adjacent layers, temperature-controlled shelf and the chamber, as shown in Figure 1. A representative pellet was considered in each layer, and heat transfer was described between the product bulk and the sublimation interface situated inside the pellet. For computational simplicity one-dimensional transfer across the slab was assumed, i.e. border effects were neglected. Spherical symmetry was assumed for the pellets, which means that gradients across the slab were considered small at the pellet diameter scale. Moreover, quasi steady state for heat and mass fluxes was considered, meaning that heat and vapor accumulation inside the product, as well as vapor flux due to desorption of unfrozen water were neglected. These are relatively common assumptions for simplified modeling of the freeze-drying process [11].

With these assumptions, heat transfer for a generic representative pellet $i$ can be written as:

$$
h_{i}^{\text {Subl Bulk }}\left(\theta_{i}^{\text {Subl }}-\theta_{i}^{\text {Bulk }}\right)+h_{i}^{\text {Bulk Bulk }}\left(\theta_{i}^{\text {Bulk }}-\theta_{i-1}^{\text {Bulk }}\right)+h_{i+1}^{\text {Bulk Bulk }}\left(\theta_{i+1}^{\text {Bulk }}-\theta_{i-1}^{\text {Bulk }}\right)=0
$$

where $\theta^{\text {Subl }}$ is the temperature at the sublimation front inside the pellet, $\theta^{\text {Bulk }}$ is the product bulk temperature and $h$ are the corresponding heat transfer coefficients, dependent on local pressure and on the sublimation front position. Vapor transfer equations were similar, with the vapor pressure as driving force. Special forms of these equations were written for the boundary pellets, at the top and the bottom of the tray.

Unavoidable coupling between heat and mass transfer occurs through the vapor equilibrium condition at the sublimation interface and through the heat balance, specifying that incoming heat flux is mainly used for ice sublimation:

$$
\begin{gathered}
P_{i}^{\text {Subl }}=P_{\text {sat }}\left(\theta_{i}^{\text {Subl }}\right) \\
h_{i}^{\text {Subl Bulk }}\left(\theta_{i}^{\text {Subl }}-\theta_{i}^{\text {Bulk }}\right)+L_{\text {subl }} k_{i}^{\text {Subl Bulk }}\left(P_{i}^{\text {Subl }}-P_{i}^{\text {Bulk }}\right)=0
\end{gathered}
$$

In these equations $P^{S u b l}, P^{B u l k l}$ and $P_{\text {sat }}$ denote vapor pressures at the sublimation interface, in the bulk of the slab and saturation pressure respectively; $k$ is the appropriate mass transfer coefficient and $L_{\text {subl }}$ the specific sublimation heat. 
The evolution of the frozen region radius $\left(r^{S u b l}\right)$ is calculated knowing the sublimation mass flux, the current sublimation area and the ice concentration in the frozen product $\left(D_{\text {Ice }}\right)$ :

$$
\frac{d r_{i}^{\text {Subl }}}{d t}=k_{i}^{\text {Subl Bulk }}\left(P_{v i}^{\text {Subl }}-P_{v i}^{\text {Bulk }}\right) /\left(4 \pi\left(r_{i}^{\text {Subl }}\right)^{2} D_{\text {Ice }}\right)
$$

To complete the model, unfrozen water desorption equations for the dry part of the pellet can be added [12].

These coupled algebraic and differential equations for all representative pellets in the tray were solved numerically using Matlab 7 simulation software (TheMathWorks, Natick, MA).

\section{Model parameter determination}

The model parameters specific to the freeze-drying apparatus, such as the heat transfer coefficient between the temperature-controlled shelf and the plate or the mass transfer coefficient between the product and the chamber, the chamber and the condenser, etc. were determined in previous lyophilization runs [12]. Product specific properties such as heat and mass transfer coefficients and desorption time constants in the dry product (inside pellets), were determined in compact layer experiments, by fitting the appropriate model [12]. The heat and mass transfer coefficients in the slab (outside pellets) were determined by fitting the duration of the primary drying, as indicated by the partial water pressure in the chamber, and the product temperature evolution, as indicated by the thermocouples placed in the slab.

\section{Results}

Preliminary model validation was performed based on freeze-drying experiments described in the Materials and Methods section. A typical result for a $45 \mathrm{~mm}$ thick slab of pellets freezedried at $35^{\circ} \mathrm{C}$ and $30 \mathrm{~Pa}$ is presented. A fair agreement between bulk temperatures measured by thermocouples placed in the product and calculated temperatures was observed (Figure 2). Exact locations of the thermocouples in the product layer were not very well controlled, so rigorous comparison is problematic, but general agreement is apparent. It should be noted that the previously developed and validated model for freeze-drying of a solution in compact layer configuration [12] was not able to predict, even qualitatively, the observed temperature evolution in a layer of pellets. In the compact layer configuration, the product temperature evolution during the primary and secondary drying phases are relatively well differentiated: in the primary drying (up to about $35 \mathrm{~h}$ in Figure 2) the product temperature is significantly lower then the shelf temperature due to heat absorbed by the ice sublimation, and it becomes 
close to the shelf temperature in the secondary drying when ice disappears. The end of the self-cooling effect of the ice sublimation is relatively clearly marked, when the sublimation front reaches the tray bottom. Moreover, top, core and bottom product temperatures are relatively close to each other, due to the high thermal conductivity of ice in the frozen layer and of the low heat flux in the dry layer.

In contrast, in a layer of pellets very high temperature gradients exist due to relatively poor thermal conductivity of the porous slab (Figure 2). Primary and secondary drying occur simultaneously at various locations. In the considered example, primary drying is essentially finished before $20 \mathrm{~h}$ at the product slab bottom, near the heat source (shelf), while ice is still present at $50 \mathrm{~h}$ at the slab top. The self-cooling effect due to ice sublimation disappears gradually, because the sublimation front area diminishes continually, as the sublimation fronts retracts towards the center of the pellets.

Partial vapor pressure measurement in the chamber gives useful complimentary information on the overall freeze-drying rate, in contrast to temperature measurement, which is limited to the immediate vicinity of the thermocouple. Again, good agreement between measured and calculated vapor pressures was found (Figure 3). Compared to the compact layer configuration, freeze-drying of pellets shows a high initial drying rate (near $10 \mathrm{~h}$, when the shelf temperature reaches its maximum) followed by a relatively quick decrease. This is due to the fact that the sublimation front moves towards the center of the pellets, its area decreases and heat and mass transfer resistances through the dry part of the pellet increase. The compact layer model predicts a relatively flat drying rate, inappropriate for pellets.

All these differences between mechanisms involved in compact layer and particle slab freezedrying support the development of a specific model for freeze-drying of pellets, since the objective is the determination of local drying history undergone by the product.

\section{Conclusions}

The paper describes a heat and mass transfer model developed specifically for freeze-drying of pellets. Local drying conditions and the overall drying rate appear to be different from freeze-drying behavior in a compact layer. Preliminary validation indicates reasonable agreement with experimental temperature and vapor pressure measurements, but further work is needed to extend and verify the validity of the model for a wide range of drying conditions, in terms of shelf temperature, total chamber pressure, product layer thickness, pellet size, product composition and structure, etc. A major challenge is the determination of pressuredependent properties such as heat and mass diffusivity inside pellets and in the porous product 
layer. Also, comparison with different modeling approaches such as [10], based on experimental validation, will shed light on the appropriate hypothesis concerning the position of the sublimation front (inside pellets vs. across the slab). The ultimate goal is reliable prediction of local temperature and vapor pressure in the product, for freeze-drying cycle development and control based on quality criteria, such as glass transition temperature and moisture content.

\section{References}

[1] Jennings, T.A., 1999, Lyophilization: Introduction and Basic Principles, Interpharm/CRC, Boca Raton.

[2] Adams, G.D.J., Ramsay, J.R., 1996, "Optimizing the Lyophilization Cycle and the Consequences of Collapse on the Pharmaceutical Acceptability of Erwinia LAsparaginase", Journal of Pharmaceutical Sciences, 85, pp. 1301-1305.

[3] Bellows, R.J., King, C.J., 1972, "Freeze-Drying of Aqueous Solutions Maximum Allowable", Cryobiology 9, pp. 559-561.

[4] Passot, S., Fonseca, F., Barbouche, N., Alarcon-Lorca, M., Rolland, D., Rapaud, M., Marin, M., 2007, "Effect of Product Temperature During Primary Drying on the LongTerm Stability of Lyophilized Proteins", Pharmaceutical Development and Technology, 12(6), pp. 543-553.

[5] Pikal, M.J., Shah, S., 1990, "The Collapse Temperature in Freeze-Drying: Dependence on Measurement Methodology and Rate of Water Removal from the Glassy State", International Journal of Pharmacy, 62, pp. 165-186.

[6] Chang, B.S., Fisher, N.L., 1995, "Development of an Efficient Single-Step Freeze-Drying Cycle for Protein Formulations", Pharmaceutical Research, 12, pp. 831-837.

[7] Pikal, M.J., 1985, "Use of a Laboratory Data in Freeze Drying Process Design : Heat and Mass Transfer Coefficients and the Computer Simulation of Freeze Drying", Journal of Parenter Science and Technology, 39, pp. 115-138.

[8] Mascarenhas, W.J., Akay, H.U., Pikal, M.J., 1997, "A Computational Model for Finite Element Analysis of the Freeze-Drying Process", Computer Methods in Applied Mechanics and Engineering, 148(1-2), pp. 105-124. 
[9] Sadikoglu, H., Liapis, A.I., 1997, "Mathematical Modelling of the Primary and Secondary Drying Stages of Bulk Solution Freeze-Drying in Trays: Parameter Estimation and Model Discrimination by Comparison of Theoretical Results with Experimental Data", Drying Technology, 15(3\&4), pp. 791-810.

[10] Liapis, A.I., Bruttini, R., 2009, "A Mathematical Model for the Spray Freeze Drying Process: The Drying of Frozen Particles in Trays and in Vials on Trays", International Journal of Heat and Mass Transfer, 52, pp. 100-111.

[11] George, J.P., Datta, A.K., 2002, "Development and Validation of Heat and Mass Transfer Models for Freeze-Drying of Vegetable Slices", Journal of Food Engineering, 52(1), pp. 89-93.

[12] Trelea, I.C., Passot, S., Fonseca, F., Marin, M., 2007, "An Interactive Tool for FreezeDrying Cycle Optimisation Including Quality Criteria", Drying Technology, 25, pp. 741-751. 
Model for heat and mass transfer in freeze-drying of pellets

\section{Figure captions}

Figure 1. Schematic representation of heat transfer during freeze-drying of pellets.

Figure 2. Model validation based on product temperature. Measured product temperature (symbols), product temperature calculated by the presented model (solid), product temperature calculated by a compact layer model (dash-dotted), shelf temperature (bold) and chamber temperature (dotted).

Figure 3. Model validation based on partial vapor pressure in the chamber. Measured vapor pressure (symbols), vapor pressure calculated by the presented model (solid), vapor pressure calculated by a compact layer model (dash-dotted), total (vapor + inert gas) pressure in the chamber (bold). 
Model for heat and mass transfer in freeze-drying of pellets

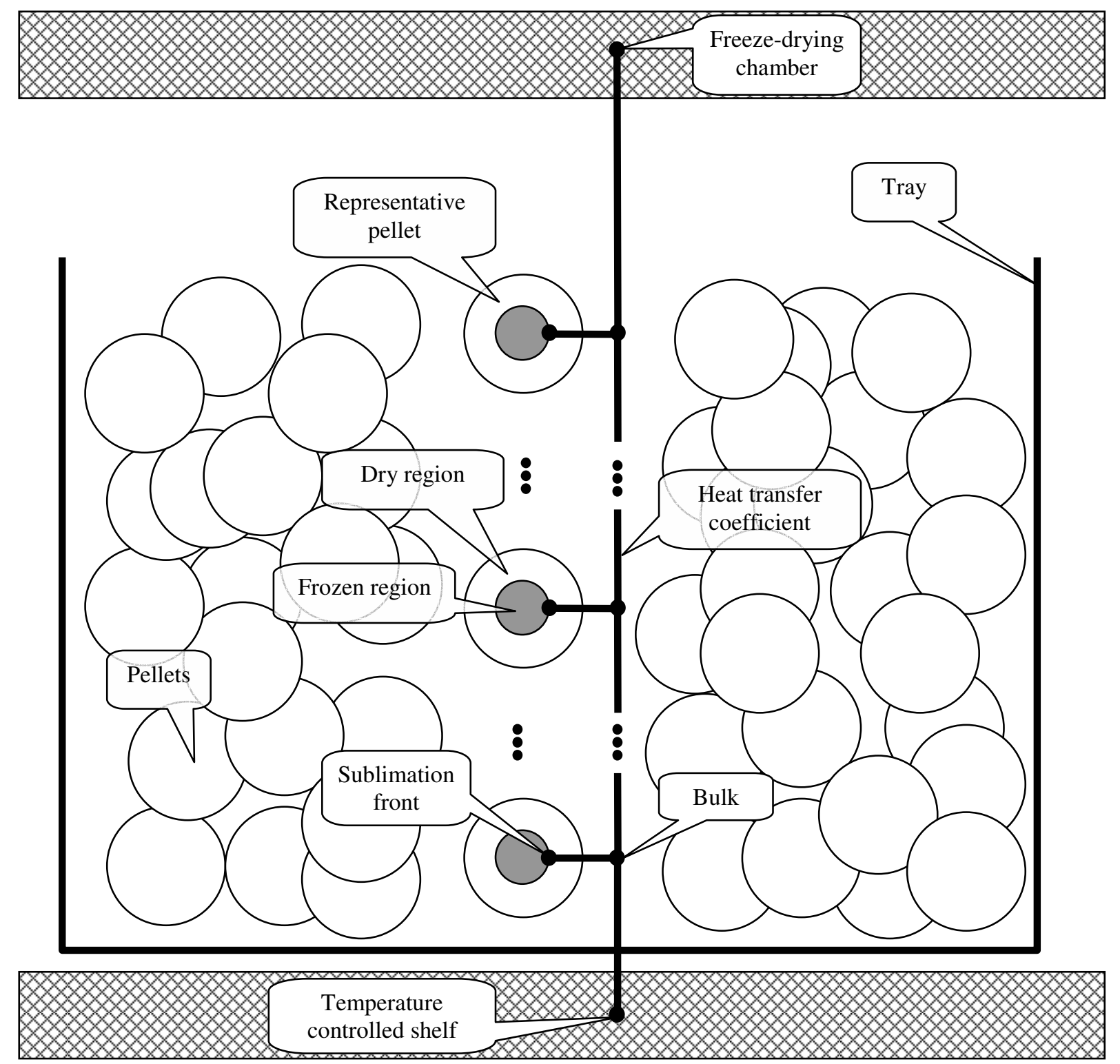

Figure 1. Schematic representation of heat transfer during freeze-drying of pellets. 
Model for heat and mass transfer in freeze-drying of pellets

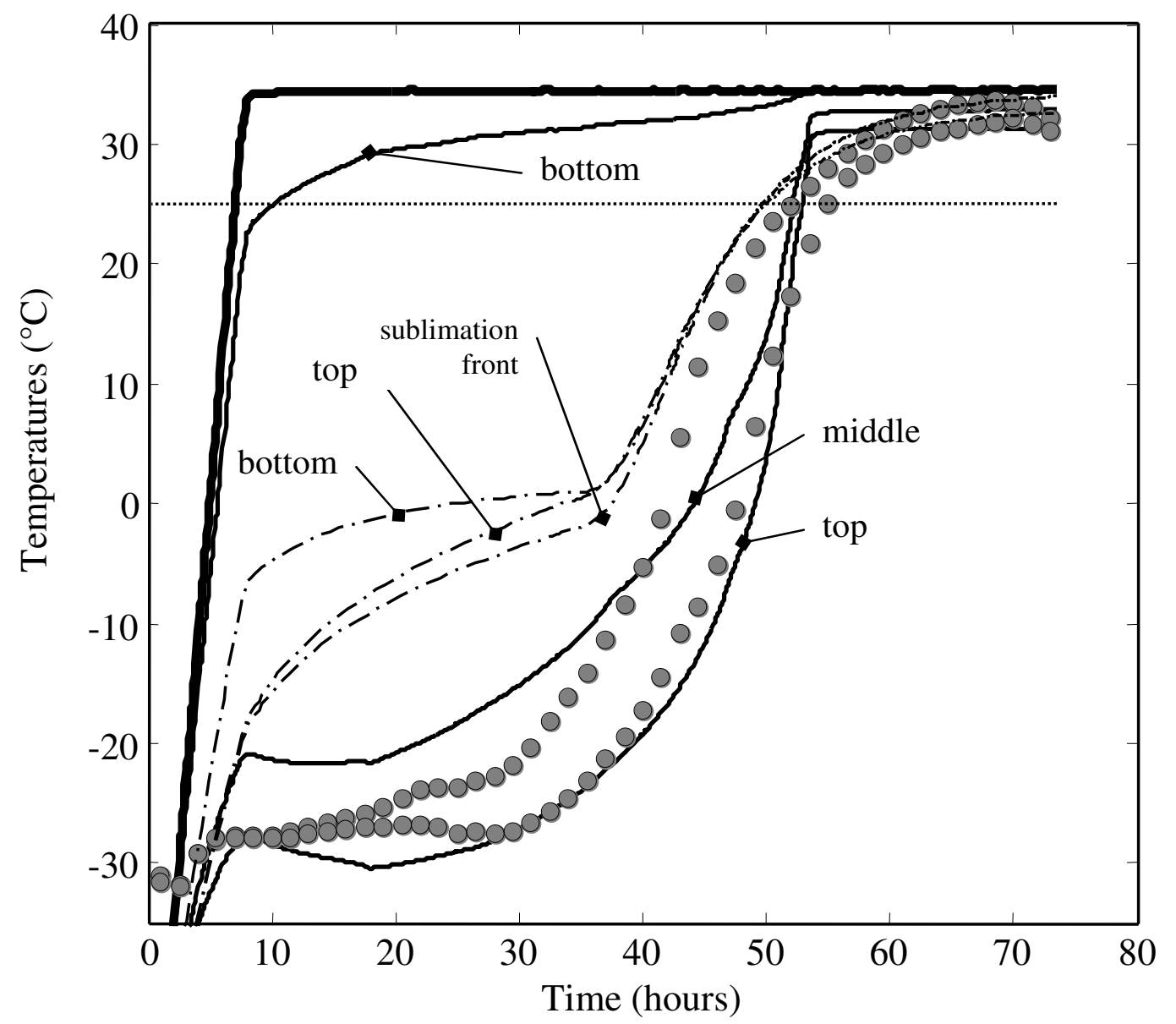

Figure 2. Model validation based on product temperature. Measured product temperature (symbols), product temperature calculated by the presented model (solid), product temperature calculated by a compact layer model (dash-dotted), shelf temperature (bold) and chamber temperature (dotted). 
Model for heat and mass transfer in freeze-drying of pellets

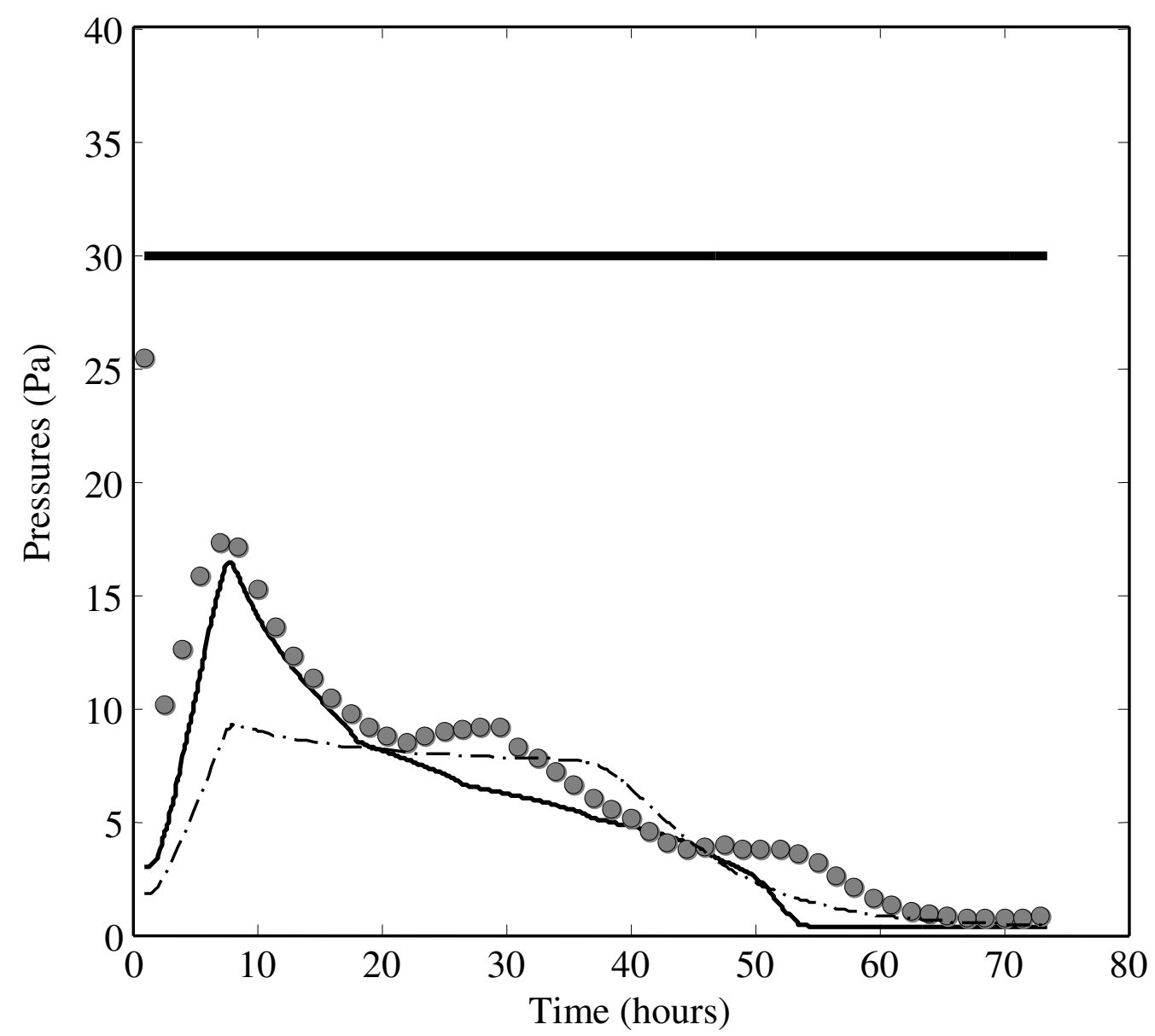

Figure 3. Model validation based on partial vapor pressure in the chamber. Measured vapor pressure (symbols), vapor pressure calculated by the presented model (solid), vapor pressure calculated by a compact layer model (dash-dotted), total (vapor + inert gas) pressure in the chamber (bold). 